\title{
Eikonal Formulation of the Minimal Principle for Scroll Wave Filaments
}

\author{
K. H.W. J. ten Tusscher ${ }^{1}$ and A.V. Panfilov ${ }^{1,2}$ \\ ${ }^{1}$ Department of Theoretical Biology, Utrecht University, Padualaan 8, Utrecht, 3584 CH, The Netherlands \\ ${ }^{2}$ Division of Mathematics, University of Dundee, 23 Perth Road, Dundee DD1 4HN, United Kingdom
}

(Received 27 November 2003; published 3 September 2004)

\begin{abstract}
Recently, Wellner et al. [Proc. Natl. Acad. Sci. U.S.A. 99, 8015 (2002)] proposed a principle for predicting a stable scroll wave filament shape as a geodesic in a 3D space with a metric determined by the inverse diffusivity tensor of the medium. Using the Hamilton-Jacobi theory we show that this geodesic is the shortest path for a wave propagating through the medium. This allows the use of shortest path algorithms to predict filament shapes, which we confirm numerically for a medium with orthotropic anisotropy. Our method can be used in cardiac tissue experiments since it does not require knowledge of the tissue anisotropy.
\end{abstract}

DOI: 10.1103/PhysRevLett.93.108106

PACS numbers: $87.19 . \mathrm{Hh}, 87.10 .+\mathrm{e}, 87.19 . \mathrm{Nn}$

Introduction.-Scroll waves have been reported in chemical systems, such as the Belousov-Zhabotinsky reaction [1], in biological systems, such as aggregations of the slime mould Dictyostelium discoideum [2], and in cardiac tissue [3,4], where they underlie life threatening cardiac arrhythmias [5]. Theoretical studies of scroll waves [6-8] have shown that their dynamics are governed by filament curvature and twist. In the case of positive filament tension, scroll rings contract and disappear, whereas nonclosed filaments attain a stable shape [6]. These stable configurations have the shape of a straight line in isotropic media but can have much more complex shapes in anisotropic media [9-11]. Predicting stable filament shapes in anisotropic media is of great importance for understanding the 3D organization of cardiac arrhythmias.

Recently, Wellner et al. proposed a "minimal principle for rotor filaments" [12]. They showed that the stable filament shape of a scroll wave in a 3D anisotropic medium is described by a geodesic in a 3D space with a metric given by the inverse diffusivity tensor of the medium, thus providing an elegant analytical formulation for predicting filament shapes. Its practical application, however, has some limitations: First, to predict filament shape, the method requires a priori knowledge of the diffusivity tensor of the medium. Furthermore, to specify boundary conditions for the geodesic equation, a priori knowledge of the position of filament ends on boundaries is required. Therefore, Wellner et al. suggest that future research in this direction should make use of variational principles in geometric contexts [12].

In this Letter, we propose an alternative geometrical formulation of the minimal principle for scroll wave filaments, based on the Hamilton-Jacobi variational approach. We show that the geodesic describing the stable scroll wave filament between two given points is the path of shortest travel time of a wave propagating between these two points in the same medium as in which the scroll wave rotates. We show that this shortest wave path can be found using standard shortest path wave algorithms, with wave propagation computed using the same set of equations as for scroll wave dynamics. Our method predicts global filament position and shape, without requiring either a priori knowledge of the position of filament ends on boundaries or of the anisotropic diffusion tensor of the medium, provided that wave arrival times can be found. This makes our method more practically suited for experiments on cardiac tissue.

Reformulation of the minimal principle.-Let us consider the following reaction-diffusion model:

$$
\frac{\partial u}{\partial t}=\frac{\partial}{\partial x_{i}} D_{i j} \frac{\partial u}{\partial x_{j}}+\Phi(u, \vec{v}) ; \quad \frac{\partial \vec{v}}{\partial t}=\vec{\Psi}(u, \vec{v}),
$$

where $D_{i j}(\vec{x})$ is the diffusivity tensor, which may depend on the local coordinates $\vec{x}=\left(x_{1}, x_{2}, x_{3}\right)$, and $\Phi(u, \vec{v}), \vec{\Psi}(u, \vec{v})$ are nonlinear functions which do not depend on $\vec{x}$ explicitly.

As stated in [12] the configuration of the filament of a stable 3D vortex in Eqs. (1) will be along a geodesic in a Riemannian space with a metric:

$$
d s=\left(G_{i j} d x_{i} d x_{j}\right)^{1 / 2} ; \quad G_{i j}=D_{i j}^{-1},
$$

where $G_{i j}(\vec{x})$ is the metric tensor (summation convention is assumed).

Note that in the formulation of (2) the dimension of the "path element" $d s$ is [time $]^{1 / 2}$, and its physical meaning is not obvious. To reformulate the dimension of the path length, first let us introduce a dimensionless metric tensor: $g_{i j}=\hat{D}_{i j}^{-1}=\frac{D_{i j}^{-1}}{D_{0}}$, where $D_{0}=1 \frac{\left[\text { space }{ }^{2}\right.}{[\text { time }]}$. Second, we introduce $v_{0}$, the velocity of wave propagation in Eqs. (1) with $D_{i j}=D_{0} \delta_{i j}$ ( $\delta_{i j}$ is the unitary tensor). Note that the choice of the value of $D_{0}$ is arbitrary; however, $v_{0}$ should be evaluated at the chosen value of $D_{0}$. Using these two new quantities, we can rewrite (2) as 


$$
d t=\frac{1}{v_{0}}\left(g_{i j} d x_{i} d x_{j}\right)^{1 / 2} ; \quad g_{i j}=\hat{D}_{i j}^{-1} .
$$

The "path length" $d t$ now has the physical dimension of [time], and as we will demonstrate, it decribes the activation time of the medium. Note that, because the governing equations of a geodesic are invariant under a constant scaling (see p. 8016 of [12]), metric (3) is equivalent to metric (2).

The geodesic $x_{i}(t)$ between two points $\vec{x}_{0}$ and $\vec{x}_{1}$ in a Riemannian space (3) minimizes the functional:

$$
I=\frac{1}{v_{0}} \int_{\vec{x}_{0}}^{\vec{x}_{1}}\left(g_{i j} \dot{x}_{i} \dot{x}_{j}\right)^{1 / 2} d t ; \quad(=d / d t) .
$$

Following the standard Hamilton-Jacobi theory [13], consider (4) with an arbitrary upper limit, i.e., introduce a function $T(\vec{X})$, which is the value of $I$ (4) along the geodesics from point $\vec{x}_{0}$ to an arbitrary point $\vec{X}$ :

$$
T(\vec{X})=\frac{1}{v_{0}} \int_{\vec{x}_{0}}^{\vec{X}}\left(g_{i j} \dot{x}_{i} \dot{x}_{j}\right)^{1 / 2} d t=\int_{\vec{x}_{0}}^{\vec{X}} F(\vec{x}, \dot{\vec{x}}) d t .
$$

A general variation of (5) is given (see, e.g., [13]) as

$$
\delta T=\frac{\partial F}{\partial \dot{x}_{i}} \delta x_{i}+\int_{\vec{x}_{0}}^{\vec{x}}\left(\frac{\partial F}{\partial x_{i}}-\frac{d}{d t} \frac{\partial F}{\partial \dot{x}_{i}}\right) \delta x_{i} d t .
$$

Since we have integrated along a geodesic, the second term in (6) equals zero. Using the symmetry of the metric tensor $\left(g_{i j}=g_{j i}\right)$, we obtain

$$
\frac{\partial T}{\partial x_{i}}=\frac{\partial F}{\partial \dot{x}_{j}}=\frac{1}{v_{0}} \frac{g_{i j} \dot{x}_{i}}{\left(g_{k l} \dot{x}_{k} \dot{x}_{l}\right)^{1 / 2}} .
$$

Substituting (7) and (3) in the following equation yields

$$
\hat{D}_{i j} \frac{\partial T}{\partial x_{i}} \frac{\partial T}{\partial x_{j}} \equiv g_{i j}^{-1} \frac{\partial T}{\partial x_{i}} \frac{\partial T}{\partial x_{j}}=\frac{1}{v_{0}^{2}} \frac{g_{i j} \dot{x}_{i} \dot{x}_{j}}{g_{k l} \dot{x}_{k} \dot{x}_{l}}=\frac{1}{v_{0}^{2}} .
$$

Equation (8) is known as the zero-order eikonal equation for wave propagation in the reaction-diffusion system (1) [14,15], where $T$ stands for the arrival time of the wave. Now we see that the value of function (4) can be interpreted as the travel time of a wave propagating from point $\vec{x}_{0}$ to $\vec{x}_{1}$. Furthermore, the geodesic, and hence the stable filament shape [12], is the path of shortest travel time of a wave propagating from point $\vec{x}_{0}$ to point $\vec{x}_{1}$.

In conclusion, we have shown that the physical interpretation of the path element of the metric space introduced in [12] is the wave travel time, and hence that the "minimal path" principle can be seen as a "shortest travel time" principle. In addition, we have shown that the eikonal equation can be used to find the shortest wave path and hence the stable filament shape. Since the eikonal equation (8) is the zero-order approximation for wave propagation in Eq. (1), we can use (1) rather than (8) to find the shortest wave path. The wave algorithms used for finding the shortest wave path are discussed below.
Shortest path wave algorithms.-To solve the shortest path problem, we use two algorithms similar to the classical Lee-Moore algorithms for finding the shortest path in a maze $[16,17]$. The principle is illustrated in Fig. 1(a). Let us denote the vertical direction in the plane with the $y$ coordinate and refer to a horizontal line of points as a $y$ layer. To find the path of shortest travel time from point $\mathrm{A}$ to the upper boundary of the plane, we initiate a wave at point $\mathrm{A}$ and find the point with the earliest arrival time at the upper boundary (point B). The shortest wave path is determined by stepwise tracing back from point $B$ to point $A$ and finding the point of earliest arrival time (black circles) in each $y$ layer and connecting these points.

To find a global shortest wave path between the lower and the upper boundaries, we modify the algorithm as follows. We initiate a wave at the whole lower boundary of the medium, rather than at a single point and find the arrival times [Fig. 1(b), continuous lines]. Then we do the same for a planar wave initiated at the upper boundary [Fig. 1(b), broken lines]. The global shortest wave path of the medium is the line connecting the points that have the minimum summed arrival time in each $y$ layer [Fig. 1(c)].

Numerical results. -We illustrate the predictive value of our wave algorithms by numerical simulations in a two variable FitzHugh-Nagumo model for excitable media of the form (1) with

$$
\begin{aligned}
& \Phi(u, \vec{v})=-k u(u-a)(u-1)-u v ; \\
& \vec{\Psi}(u, \vec{v})=\epsilon(k u-v) .
\end{aligned}
$$

Parameter values are $a=0.07, k=8$, and $\epsilon=0.066$.

We study three different cases of anisotropy. In the first case we use a conductivity tensor $D_{1}$ with parallel curved fiber organization similar to that of [11] but extended to the orthotropically anisotropic case [18]: $D_{x x}=D_{T}$; $D_{y y}=D_{L} \cos (\alpha)^{2}+D_{T T} \sin (\alpha)^{2} ; D_{y z}=D_{z y}=\left(D_{L}-\right.$ $\left.D_{T T}\right) \cos (\alpha) \sin (\alpha) ; \quad D_{z z}=D_{L} \sin (\alpha)^{2}+D_{T T} \cos (\alpha)^{2} ;$ $D_{x y}=D_{x z}=D_{y x}=D_{z x}=0$, where $D_{L}, D_{T}$, and $D_{T T}$ are the conductivities parallel and transversal to the fibers, and transversal to the plane in which the fibers
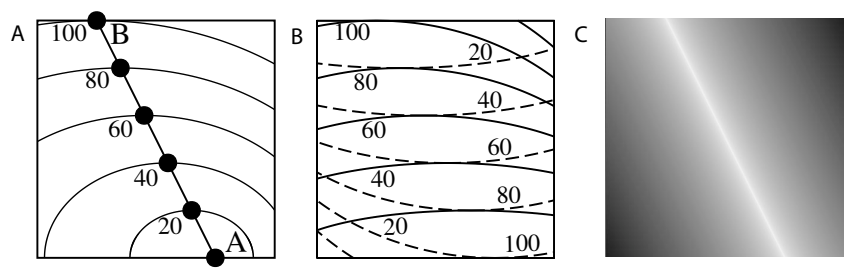

FIG. 1. Illustration of the first (a) and second (b),(c) shortest path wave algorithm. In (a) and (b) lines with numbers are isoarrival time lines. In (b) the continuous lines are for an upward and the broken lines for a downward propagating wave. In (c) the grayscale indicates summed arrival time (white is short; black is long). For an explanation of the algorithms, see the text. 
are organized, respectively. We choose $D_{L}=1, D_{T}=$ $1 / 4$, and $D_{T T}=1 / 16 . \alpha$ is a function of position in the $y$ direction: $\tan (\alpha)=\frac{2 \pi A}{N} \cos \left(\frac{2 \pi y}{N}\right), A=20$.

For the second and third cases we performed the following transformations of the original conductivity tensor:

$$
D_{2}=\mathbf{A}^{T} D_{1} \mathbf{A} ; \quad D_{3}=\mathbf{B}^{T} D_{2} \mathbf{B} .
$$

Matrix A performs a rotation in the $y z$ plane with an angle $\beta$, with $\beta=2(N / 2-z)$. Matrix $\mathbf{B}$ performs a rotation in the $x y$ plane by an angle $\gamma=2(N / 2-x)$.

Computations were performed using an explicit time integration scheme on an $N \times N \times N$ array, with $N=$ 120 and using Neumann boundary conditions. The time integration step was $d t=0.02$; the space integration step was $d x=0.01$. We used initial data corresponding to a broken wave front to create scroll waves.

We start with a medium described by conductivity tensor $D_{1}$. Figure 2(a) shows a stable scroll wave rotating in this medium. The scroll wave filament, together with the prediction we found using the first wave algorithm [Fig. 1(a)], by initiating a wave at the point were the scroll filament touches the $y=0$ boundary, is shown in Fig. 2(b). We see that our algorithm's prediction agrees well with the actual filament shape (note that the resolution of our algorithm is restricted to the space step of the simulation grid).

Because of the translational symmetry of $D_{1}$ in the $x z$ plane, filaments with similar shapes can be obtained starting from almost any point at the $y=0$ boundary, provided it is not too close to the other boundaries. To unfold this degeneracy, we performed simulations in a medium with conductivity tensor $D_{2}$, obtained via a nonuniform rotation of tensor $D_{1}$ in the $y z$ plane. To determine the global shortest wave path of this medium we applied the second wave algorithm [Fig. 1(b)], with plane waves initiated at the $y=0$ and $y=N$ boundaries.

We found a family of shortest wave paths making up a shortest path surface (Fig. 3 gray) that is translationally invariant in the $x$ direction. We tested the prediction from the wave algorithm by initiating two scroll waves at
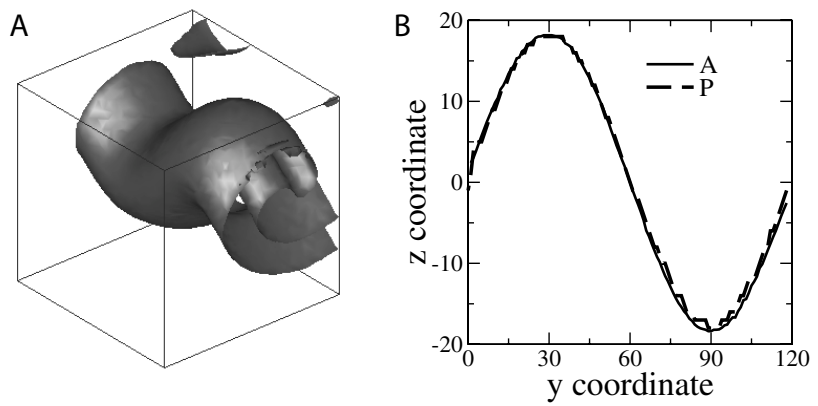

FIG. 2. (a) Scroll wave in a medium with conductivity tensor $D_{1}$. (b) Actual (A) and predicted (P) filament shape. different initial positions. Figures 3(a)-3(d) show that the two scroll wave filaments (both in black) align with the predicted shortest wave path surface in the course of time, each at a different $x$ position.

To unfold the symmetry of conductivity tensor $D_{2}$ in the $x$ direction, we performed simulations in a medium with conductivity tensor $D_{3}$ (obtained via a nonuniform rotation of tensor $D_{2}$ in the $x y$ plane). Figure 4 shows the shortest wave path (gray) predicted by the second wave algorithm, which now is a single curve that acts as a global attractor for all filaments running from $y=0$ to $y=N$. Figures 4(a) -4 (d) show that the filament (black) of a scroll wave initiated at an arbitrary initial position indeed aligns with the predicted shortest wave path curve in the course of time.

Discussion.-The original Wellner et al. [12] principle and our reformulation imply that the filament shape of a stable scroll wave in a 3D anisotropic reaction-diffusion medium is solely defined by the diffusion tensor and does not depend on the reaction kinetics of the medium. In our view this is similar to previous observations that filaments in rectangular isotropic media minimize their length and attain a straight shape independent of the medium's reaction kinetics. In the anisotropic case, where shortest length is not clearly defined, the minimal principle states that filaments will attain the shape of the path of shortest travel time. We demonstrated that this shortest travel time path can be found with wave algorithms that use the same equations for wave propagation as those used for the actual scroll wave simulations. Note that this by no means implies any similarity between equations describ-
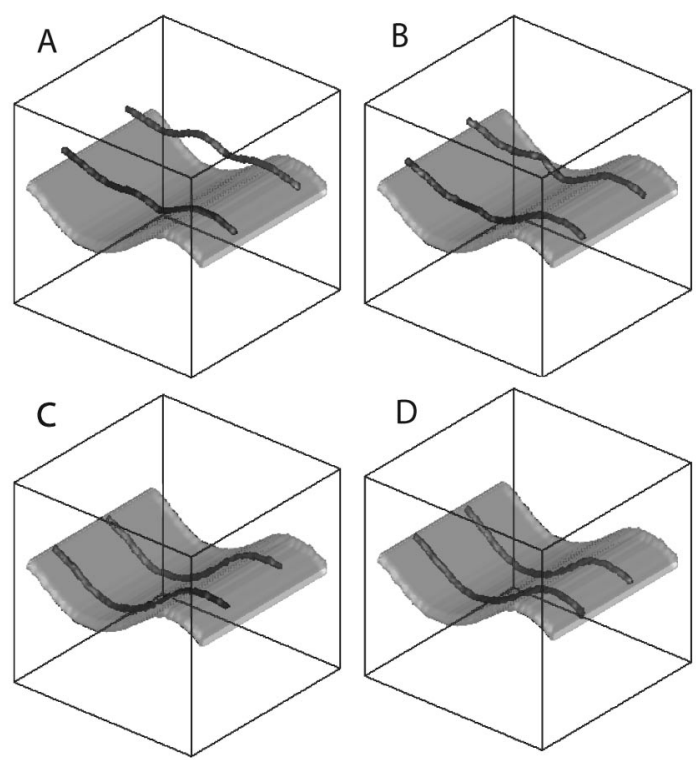

FIG. 3. Convergence of two filaments (both in black) to the shortest wave path predicted by our algorithm (gray). Snapshots were taken at time (dimensionless units) 23.5 (a), 50 (b), 850 (c), and 3100 (d). 


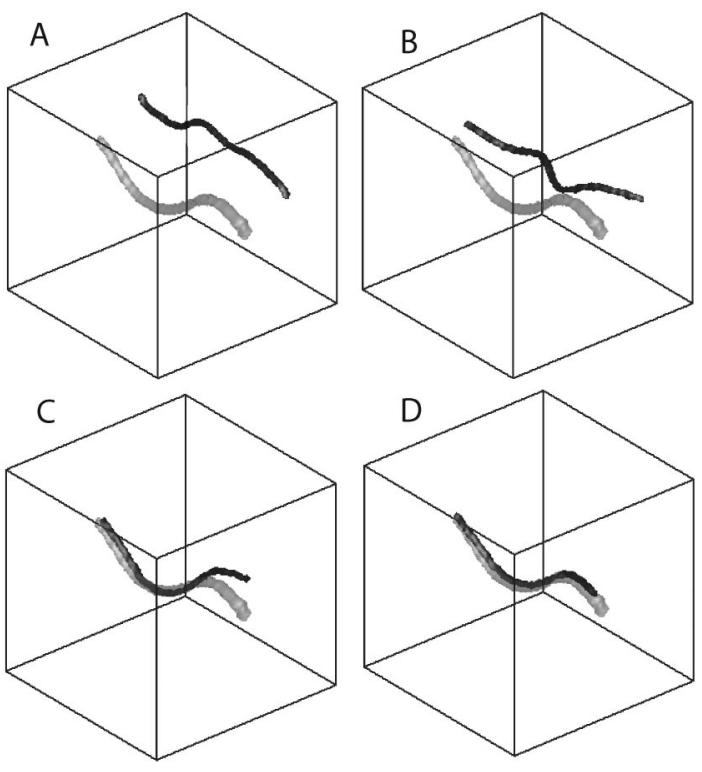

FIG. 4. Convergence of a scroll wave filament (black) to the shortest wave path predicted by our algorithm (gray). Snapshots were taken at time 23.5 (a), 100 (b), 850 (c), and 3100 (d).

ing filament dynamics (which are still unknown for general anisotropic media) and equations describing wave propagation.

Note, however, that these explanations are only qualitative. Further research is needed to establish why stable filament shape does not depend on reaction kinetics and to determine why the filament of a scroll wave in an anisotropic medium follows the path of shortest travel time of a wave propagating through the medium.

In this Letter, we propose an eikonal formulation of the minimal principle for scroll wave filaments which allows the application of shortest path wave algorithms to find stable filament configurations. An important feature of this method is that it allows the prediction of stable filament shapes without a priori knowledge of the anisotropic conductivity tensor of the medium and of the filament end positions on the tissue boundaries. The only information that is required are the arrival times of a planar wave front initiated at the tissue boundaries. The method can be applied to any tissue anisotropy and boundary geometry. Since arrival times may be obtained for $3 \mathrm{D}$ wave propagation in cardiac tissue, using, for example, the transillumination technique [19], our method can thus be applied to real cardiac tissue.

The original formulation of the minimal principle [12] was proposed for the case of isotropic transversal anisotropy. Here, we extended the numerical verification of this principle to media with orthotropic anisotropy, which is extremely relevant for cardiac tissue [18].

Our method has several limitations which should be addressed in future studies: First, our wave algorithm, as well as the original geodesic formulation, is proposed for the case of homogeneously excitable media and nonmeandering scroll waves. Second, our wave algorithm finds a global shortest wave path, while filaments can potentially be stable at local minima that may exist for particular anisotropy configurations. Third, our method is precise only if one uses the eikonal equation (8) in the shortest path wave algorithms. The approximation used in this Letter [Eqs. (1)] is accurate provided that the effect of curvature on wave propagation is small.

Conclusion.-We propose an eikonal formulation of the minimal principle for stable filament shapes which allows us to effectively predict filament configurations in complex anisotropic excitable media.

We are grateful to Dr. S. Fomel and Dr. R. Hinch for valuable discussions on important aspects of our Letter. This research is funded by the Netherlands Organization for Scientific Research (NWO) through Grant No. 620061351 of the Research Council for Physical Sciences (EW) (K. H.W. J. t. T.).

[1] A. T. Winfree, Science 181, 937 (1973).

[2] F. Siegert and C. J. Weijer, Proc. Natl. Acad. Sci. U.S.A. 89, 6433 (1992).

[3] P. S. Chen et al., Circ. Res. 62, 1191 (1988).

[4] I. Efimov, V. Sidorov, Y. Cheng, and B. Wollenzier, J. Cardiovasc. Electrophysiol. 10, 1452 (1999).

[5] R. A. Gray and J. Jalife, Chaos 8, 65 (1998).

[6] A.V. Panfilov and A. N. Rudenko, Physica (Amsterdam) 28D, 215 (1987).

[7] J. P. Keener, Physica (Amsterdam) 31D, 269 (1988).

[8] S. Setayeshgar and A. J. Bernoff, Phys. Rev. Lett. 88, 028101 (2001).

[9] O. Berenfeld and A. M. Pertsov, J. Theor. Biol. 199, 383 (1999).

[10] M. Wellner, O. Berenfeld, and A. M. Pertsov, Phys. Rev. E 61, 1845 (2000).

[11] O. Berenfeld, M. Wellner, J. Jalife, and A. M. Pertsov, Phys. Rev. E 63, 061901 (2001).

[12] M. Wellner, O. Berenfeld, J. Jalife, and A. Pertsov, Proc. Natl. Acad. Sci. U.S.A. 99, 8015 (2002).

[13] V. I. Smirnov, A Course on Higher Mathematics (Pergamon Press, New York, 1964).

[14] P. Franzone, L. Guerri, and S. Tentoni, Math. Biosci. 101, 155 (1990).

[15] J. Keener and J. Sneyd, Mathematical Physiology (Springer-Verlag, New York, Heidelbeg, Berlin, 1998).

[16] E. Moore, Annals Comp. Lab. Harvard Univ. 30, 285 (1959).

[17] V. I. Krinsky, V. N. Biktashev, and I. R. Efimov, Physica (Amsterdam) 49D, 247 (1991).

[18] I. J. LeGrice, B. H. Smaill, L. Z. Chai, S. G. Edgar, J. B. Gavin, and P. J. Hunter, Am. J. Physiol. 269, H571 (1995).

[19] A. Pertsov, S. Mironov, A. Zaitsev, and J. Jalife, Circulation 100, I (1999). 